\title{
SEO y comunicación académica: ¿nuevo ámbito competencial y de estudios?
}

\author{
LLUÍS CODINA (Iluis.codina@upf.edu)Twitter: https://twitter.com/lcodina/ \\ CARLOS LOPEZOSA (carlos.lopezosa@upf.edu)Twitter: https://twitter.com/carloslopezosa/ \\ Profesores de la Universitat Pompeu Fabra y de la UPF Barcelona School of Management
}

\section{Introducción}

El SEO académico es el conjunto de teorías y prácticas aplicadas a optimizar la visibilidad de la comunicación académica. A su vez, podemos definir la comunicación académica como el conjunto de producciones, en general de tipo bibliográfico (artículos, libros y otros formatos), mediante el cual la comunidad científica informa sobre el progreso de la ciencia y sobre los resultados de sus investigaciones.

La oportunidad, y a la vez, la necesidad del SEO académico o ASEO (por sus siglas en inglés, esto es, Academic Search Engine Optimization) procede de una serie de factores y tendencias confluyentes, entre las que se pueden citar las siguientes:

1. Las publicaciones abiertas, como parte muy importante de la ciencia abierta.

2. Una creciente incorporación de nuevos actores al sistema de la ciencia, y de la mano de estos, a la comunicación académica.

3. La tendencia a considerar el impacto, y no solamente la publicación, como elemento privilegiado de evaluación de la ciencia y de las carreras académicas.

4. La tendencia a considerar el impacto social, y no solamente el académico, como parte fundamental de la forma de evaluar el éxito de proyectos e investigaciones.

El tercer y cuarto factor que hemos mencionado no son, ni mucho menos, nuevos, pero forman parte del grupo de tendencias porque en ambos casos su importancia se ha acentuado enormemente. De manera que se unen de forma decisiva a los otros dos que proceden de la enorme capacidad disruptiva de internet. La comunicación académica, por su parte, es un ecosistema complejo que incluye diversos actores, cuatro de los principales (pero no los únicos) son los siguientes:

- Autores e investigadores

- Grupos, departamentos o equipos de investigación

- Editoriales de revistas y libros académicos

- Universidades y centros de investigaciones

Todos ellos necesitan el SEO académico en la medida que, en su momento, el comercio electrónico necesitó el SEO convencional, o los medios de comunicación el SEO periodístico, por mencionar dos casos destacados en los que se generó una nueva demanda de profesionales cualificados.

Bajo el principio de que el acceso abierto a los resultados de investigación acelera el avance del conocimiento, todos los contenidos de la edición electrónica de CLIP se distribuyen bajo una licencia de uso y distribución Creative Commons Reconocimiento-NoComercialCompartirlgual 3.0 España (CC BY-NC-SA 3.0 ES). 


\section{¿Un nuevo ámbito que requiere nuestra atención?}

La idea fundamental que queremos presentar en este artículo es que los profesionales de la información y la documentación (o de la biblioteconomía-documentación) forman el colectivo mejor preparado para este ámbito competencial que, además, sin duda es también un ámbito de estudio.

Un nuevo ámbito competencial significa un nuevo posible perfil profesional, lo que significa a la vez posibilidades vinculadas tanto la creación de nuevos puestos de trabajo como con la mejora de los existentes.

Por su parte, un nuevo ámbito de estudio también aporta oportunidades para investigadores y académicos de nuestro ámbito, por eso parece importante considerar lo mejor posible el alcance del SEO académico.

Una razón a favor de considerar al ASEO como parte de los diferentes perfiles de la documentación (o de la biblioteconomía-documentación) es que, además de competencias en SEO, un ámbito donde en realidad convergen profesionales con muy diferentes procedencias, se necesitan competencias en comunicación académica. En este último, realmente el colectivo mejor preparado (y volvemos a decir, posiblemente el único, por ahora) es de los profesionales de nuestro ámbito.

Los diferentes aspectos, dimensiones, implicaciones, actores, etc., de la comunicación académica solamente son bien comprendidos, de una forma holística, por profesionales y estudiosos de nuestro sector. Una enumeración de algunas de sus facetas puede bastar:

- El mundo de la publicación científica (estructura de un artículo, naturaleza de las revistas académicas, funcionamiento del sistema peer review, etc.)

- El mundo de los buscadores académicos: sus diferentes clases, modos de indexación, búsqueda, etc., así como su relación con los autores: perfiles, identidad única, etc.

- El mundo de las bases de datos y otros sistemas de información académica: tipos de bases de datos, formas de acceso y explotación, sistemas de análisis, etc., e igualmente el aspecto de los perfiles y la identidad única, con elementos como ORCID, etc.

- El ámbito de los indicadores de impacto: Journal Impact Factor, CiteScore, Scimago, etc.

- El ámbito de los author services, tanto en empresas independientes (p.e. Enago. Kudos, etc.), como en editoriales académicas.

- Aspectos de factores de éxito en la publicación académica: optimización del texto, de los contenidos multimedia, claves de un buen resumen, etc.

- Y, por último, pero sin ánimo de cerrar la enumeración de temas, las complejidades y sutilezas de la ciencias abierta, la publicación abierta, los repositorios, las redes sociales académicas, etc.

Ante un ecosistema así, es la razón por la cual los autores de esta aportación al Clip, creemos que el SEO académico puede ser un nuevo perfil profesional especialmente idóneo para los profesionales y estudiosos de nuestro ámbito.

Una primera aproximación la tenemos en el nuevo rol que las bibliotecas universitarias de las mejores universidades del mundo están teniendo en la promoción y difusión de la producción bibliográfica de sus profesores.

Este, por tanto, el de los servicios para autores que están asumiendo las bibliotecas universitarias con sus políticas alrededor de portales de producción científica o repositorios es un indicador muy claro de la potencia que nuestro sector tienen en el SEO académico.

Lo que ahora está circunscrito, y de forma incipiente, en las bibliotecas universitarias, más necesarias que nunca, por cierto, gracias a estos nuevos roles, puede expandirse a los otros actores del ecosistema de la comunicación académica. 


\section{Fundamentos del SEO académico}

Parece obligado en una contribución como esta, intentar ir más allá de las declaraciones anteriores e intentar presentar un panorama del SEO académico. Cabe señalar que, seguramente, hay tantas posibilidades de interpretación del ASEO como autores. La razón (más allá de la diversidad humana) es que cada autor puede hablar desde un marco de referencia distinto: la de una biblioteca universitaria, la de una editorial académica, la de un grupo de investigación, la de un profesional de la gestión y la evaluación de la ciencia, etc.

El marco de referencia que nosotros tenemos es el de autores de producción académica, esto es de producción bibliográfica de la que estamos obligados a perseguir no solo su calidad intrínseca, sino también un razonable impacto académico y, a poder ser, social.

A partir de los sesgos inevitables que nuestro marco de referencia nos impone, podemos establecer que el objetivo principal del SEO académico es el aumento de la visibilidad de la producción académica.

Ahora bien, igual que en el SEO general el objetivo real no es el tráfico web, sino las llamadas conversiones, podemos decir que el objetivo último o real del SEO académico no es la visibilidad, en sí misma, sino incrementar, por un lado, el impacto social, y por otro, el impacto académico.

En síntesis, el SEO académico persigue incrementar:

- la visibilidad de los trabajos académicos y, como consecuencia,

- su impacto académico,

- su impacto social.

Ahora bien, mientras que el impacto académico está relativamente bien determinado, el impacto de la ciencia puede ser de tipo social o de tipo académico (propiamente dicho). El impacto social es específico de cada trabajo. Si una investigación, por ejemplo, descubre una relación causal entre determinados hábitos y una mejora de la salud, es evidente que el impacto social perseguido es el de un mayor bienestar de la población.

Existen varias disciplinas que se ocupan del impacto social de las investigaciones científicas. Una de ellas, es la que persigue mejorar la comunicación de los resultados científicos para acercarlos más al gran público.

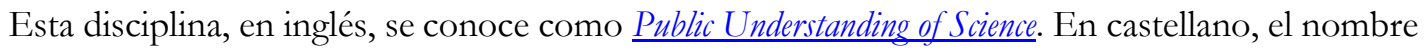
parece muy polisémico, aunque tiene un sentido mucho más preciso de lo que sugiere: Comunicación Cientifica. El Observatorio de la Comunicación Científica, es un ejemplo de excelencia en la investigación este ámbito.

Algunas variedades de las Ciencias Sociales, como la Economía de la Ciencia, también estudian los efectos de los descubrimientos científicos y su papel en el desarrollo. Con la Sociología de la Ciencia y la $\underline{T e c n o l o g i ́ a}$, por su parte, sucede algo parecido pero trasladado al estudio que los efectos de la ciencia tienen en las sociedades.

\section{Impacto académico}

Por su parte, el impacto académico consiste en incrementar las posibilidades de que un trabajo académico sea leído por otros investigadores y, como consecuencia, sea citado. Por tanto, consiste en ayudar a mejorar las métricas académicas, tanto del propio articulo (número de citas) como del autor (índice h).

Claro que, en ambos casos, necesitamos que se cumpla el primer objetivo (visibilidad), pero es muy importante tener clara la diferencia, ya que, si únicamente nos importara la visibilidad, algunas partes del SEO académico, notablemente, la identidad digital (lo veremos más adelante), carecería de sentido. 
Dado que el impacto social es diferente para cada tipo de investigación, y que incluye una amplia variedad de aspectos, estudiados por diferentes disciplinas, se suele considerar, al menos desde el marco de referencia de los autores, que:

- la misión preferente del SEO académico es el aumento de la probabilidad de ser citado. En lo que sigue, nos centraremos exclusivamente en el impacto académico de la visibilidad.

\section{PRE vs POST}

Sea como sea, debemos señalar que hay al menos dos momentos teóricos de intervención del SEO académico, a saber, antes de la publicación (esto es, durante las diversas fases de redacción) y una vez que el artículo ha sido publicado:

- Pre publicación. Esta fase interviene durante la producción del trabajo académico, típicamente durante la redacción de un artículo de revista. Podría equipararse con el SEO OnPage, en el sentido que se refiere a optimizar el contenido del artículo pensando en la indexación y la interpretación del contenido del mismo por parte de los buscadores académicos. En esta fase, se trata de optimizar el uso de palabras clave en el título, en las keywords, en el resumen y en determinados lugares del cuerpo del artículo, como en las tablas, pies de ilustraciones, títulos de las secciones, etc. De esta fase dependerá que el artículo sea filtrado y considerado relevante para determinadas búsquedas, justo aquellas que utilicen esas palabras clave. (En este punto, una aclaración tal vez innecesaria es que este concepto es independiente de los así llamados preprints que hacen referencia a otro aspecto de las publicaciones académicas, independiente del SEO -ver anexo-).

- Post publicación. Por tanto, una vez el trabajo ha sido producido y hecho público de alguna forma. Podría equipararse con el SEO OffPage. Consistiría en promover la máxima difusión del artículo sobre la base de archivarlo en repositorios. También en utilizar las redes sociales, tanto académicas como convencionales y proporcionarle así la máxima visibilidad posible.

- Otras acciones adicionales dependerán de las posibilidades del autor o del grupo de investigación, así como del posible soporte que tenga de su institución (p.e. de la biblioteca universitaria): publicar reseñas en el portal del grupo, p.e., incluirlo en newsletters, etc. De toda esta visibilidad, en teoría, dependerá el aumento de la probabilidad de citación.

Es fácil ver que hay dos momentos principales: la publicación en abierto y la difusión activa en el SEO Académico, cosa que intentamos reflejar en la lista ordenada siguiente:

1. Publicación:

- open access

- autoarchivo

2. Difusión activa

- redes sociales académicas

- redes sociales convencionales

- acciones complementarias

La publicación en abierto, ya sea mediante la publicación en modo open access o mediante el autoarchivo, conlleva una difusión implícita, podemos decir de tipo pasivo: por el mero hecho de subir un documento a un repositorio (o por haber sido publicado en open access), tal documento goza de una visibilidad potencial al poder ser indexado por los buscadores o simplemente por estar disponible en un sitio web que tiene un determinado tráfico diario.

Por eso hemos añadido la idea de una difusión activa, que consiste en el uso de redes sociales académicas, redes sociales convencionales y cualquier otra clase de acciones complementarias, como la publicación de reseñas en blogs, etc. 


\section{Acciones complementarias}

Las acciones complementarias de refuerzo (Mari Vàllez, 2016) se refieren a cualquier actividad que ayude a dar a conocer la publicación: puede ir desde poner un post en el blog personal del autor, o en la página del grupo de investigación, hasta enviar notas de prensa a los medios de comunicación o revistas especializadas, en concreto si conseguimos la ayuda del departamento de comunicación o del gabinete de prensa de nuestra institución.

Las principales editoriales académicas están tomando cada vez más en serio este aspecto, y suelen tener páginas web donde proponen o aconsejan acciones de promoción y difusión complementarias por parte de los autores, aparte las que ellas mismas, en el mejor de los casos, llevan a cabo, muchas veces desde sus plataformas digitales.

Otras acciones que se pueden llevar a cabo están limitadas solamente por la imaginación: algunas editoriales sugieren a los autores escribir reseñas de sus obras en plataformas tipo blog que ponen a su disposición. Otra estrategia puede consistir en contactar y facilitar una copia del artículo (si es el caso) a los autores citados en el mismo, etc.

Forma parte de estas acciones, la monitorización o seguimiento del artículo, que podemos hacer a través de recursos como Mendeley, Kudos o Impactstory, así como en las plataformas de las propias editoriales en la mayoría de los casos.

Publicar no es fácil (olvidemos los predatory publishers), pero atraer citas aún es más difícil. Sin embargo, cada vez más los autores son evaluados no solamente con base a su capacidad para publicar artículos, sino de su capacidad para ser un autor citado.

De hecho, hay al menos un tipo de evaluación (en España) que depende casi exclusivamente de las citaciones (no del número de publicaciones), se trata de las evaluaciones denominadas tramos de investigación o «sexenios», donde el investigador tiene que argumentar los indicadores de calidad de sus publicaciones en base, principalmente, a las citaciones recibidas.

Al mismo tiempo, el número de publicaciones no deja de crecer, entre otras cosas porque afortunadamente el número de autores en condiciones de hacerlo también crece. Naciones enteras de Asia, América y África que antes estaban prácticamente fuera del sistema de la ciencia, ahora aportan una importante producción regular de artículos científicos de gran calidad en revistas indexadas.

Una forma de conseguir visibilidad para nuestros trabajos y aumentar así la probabilidad de ser citados es aplicar los principios del SEO Académico que hemos presentado aquí. Por ello, su conocimiento es esencial por parte de investigadores, muy especialmente de aquellos que están en el inicio de su carrera académica. No obstante, cada vez es más necesario en casi cualquier momento de la misma y en cualquier nivel de agregación: Grupo de investigación, Departamento, Universidad, etc.

\section{Conclusiones}

En otras ocasiones, hemos podido ver de qué manera aspectos competenciales y ámbitos de trabajo dispersos han conducido a la consolidación de un perfil muy determinado que ha ayudado a la creación de oportunidades profesionales, así como ha propiciado ámbitos de investigación. Los ejemplos que tenemos en mente consisten en perfiles como el de la curación de contenidos, la gestión de la comunidad (community management) o el de SEO para ámbitos muy concretos, como el SEO de sitios intensivos en contenidos.

Podría ser que el ASEO o el SEO académico sea uno de estos perfiles, con capacidad, como hemos señalado para crear puestos de trabajo, o al menos, para mejorar la cualificación de puestos de trabajo. Y sin duda, es también un ámbito de investigación. Cualquier de ellas por separado mecería nuestra atención, pero unidas lo hacen todavía más. 
Otro indicador que queremos destacar es el nuevo rol que están teniendo las bibliotecas o los CRAI en las universidades. Seguramente, la biblioteca (o el CRAI) es uno de los organismos más apreciados por parte de la comunidad universitaria. Ello es debido a la continuada reinvención que las bibliotecas universitarias han experimentado en las últimas dos décadas. Como sea, buena parte de su excelente consideración en el ecosistema universitario procede sin duda de sus labores "tradicionales" que siguen siendo imprescindibles, pero sin duda, también buena parte procede del hecho de haber incorporado labores que nosotros identificamos como parte del SEO académico. Esta situación sin duda se puede extrapolar a otros ámbitos del ASEO, como las editoriales o los grupos y proyectos de investigación.

Como sea, creemos que vale la pena que nuestro ámbito, empiece a considerar seriamente las potenciales crecientes del SEO académico, tanto para la profesión como para los nuevos ámbitos de estudio.

\section{Para saber más}

Para este trabajo, no presentaremos una bibliografía convencional. Como sector emergente, tiene aún poca producción académica, al menos si pensamos en producción que utilice el término SEO académico. Como ámbito competencial hay más fuentes si acudimos a informes y propuestas no procedentes de revistas académicas convencionales. No obstante, por supuesto, hay investigaciones y publicaciones académicas, y algunas las hemos recogido. Como sea, preferimos llamar a este apartado "Para saber más", y lo hemos organizado como mostramos a continuación.

\section{Algunas referencias seleccionadas}

- Beel, J.; Gipp B.; Wilde, E. «Academic Search Engine Optimization (ASEO): Optimizing Scholarly Literature for Google Scholar and Co». Journal of Scholarly Publishing, 41 (2): 176-190, January 2010.

- Beel, J.; Gipp, B. Academic search engine spam and Google Scholar's resilience against it. J. Electron. Publ. 2010, 13, 1-28

- Codina, L. SEO académico Significación, componentes y fases. 2019

- De Gruyter Open. Why and how should you optimize academic articles for search engines?

- Elsevier. Get noticed (pdf) Sept. 2016.

- Elsevier. Get you and your research noticed!. Elsevier Publishing Campus.

- Martín-Martín, A.; Ayllón, J.M.; Orduña-Malea, E.; López-Cózar, E.D. Google Scholar Metrics Released: A Matter of Languages and Something Else. EC3 Working Papers; EC3: Granada, Spain, 2016; pp. 1-14. https://arxiv.org/abs/1607.06260v1

- Muñoz-Martín, B. Incrementa el impacto de tus artículos y blogs: De la invisibilidad a la visibilidad. Rev. Soc. Otorrinolaringol. 2015, 6, 6-32. http://hdl.handle.net/10366/126907.

- Orduña-Malea, E.; Martín-Martín, A.; Ayllon, J.M.; Delgado-Lopez-Cozar, E. The silent fading of an academic search engine: The case of Microsoft Academic Search. Online Inf. Rev. 2014, 38, 936-953. https://riunet.upv.es/bitstream/handle/10251/82266/silent-fadingmicrosoft-academic-search.pdf

- Rovira, C.; Guerrero-Solé, F.; Codina, L. Received citations as a main SEO factor of Google Scholar results ranking. El Profesional de la Información 2018, 27, 559-569

- Rovira, C.; Codina, L.; Guerrero-Solé, F.; Lopezosa, C. Ranking by Relevance and Citation Counts, a Comparative Study: Google Scholar, Microsoft Academic, WoS and Scopus. Future Internet, 2019

- UCLA Library. SEO for Authors: A How-to Guide.

- The Open University. Using Search Engine Optimization (SEO) for research publications. 
- Vàllez, Mari. Como aumentar la visibilidad de los artículos científicos (presentación). Barcelona: UOC, 2016

- Ziakis, C.; Vlachopoulou, M.; Kyrkoudis, T.; Karagkiozidou, M. Important Factors for Improving Google Search Rank. Future Internet 2019, 11, 32.

Sitios web de las principales editoriales con consejos de promoción para sus autores

- Elsevier: Sharing and promoting your article

- Emerald: Impact of Research

- SAGE: Promote Your Article

- Springer: After Publication

- Taylor and Francis: Ensuring your research makes an impact

- Wiley: Promotional Toolkit

\section{Autores}

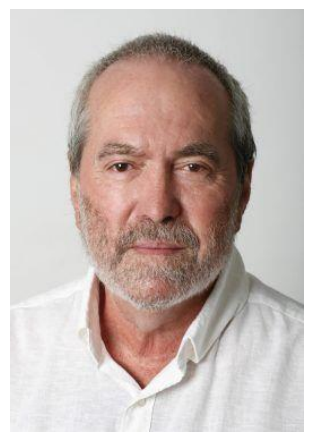

LLUÍS CODINA

Profesor de la Universitat Pompeu Fabra y de la UPF Barcelona School of Management

- Email:1luis.codina@upf.edu

- Twitter: https://twitter.com/lcodina/

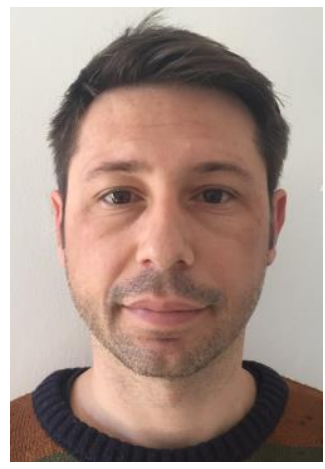

CARLOS LOPEZOSA

Profesor de la Universitat Pompeu Fabra y de la UPF Barcelona School of Management

- Email: carlos.lopezosa@upf.edu

- Twitter: https://twitter.com/carloslopezosa/ 